\title{
Exploring Differences in Androgen Deprivation Therapy Use for Prostate Cancer Between Black Men and White Men
}

\author{
Sally L. Maliski, PhD, RN, FAAN \\ Amy Garcia, DNP, FAAN \\ Ellen Harper, PhD, RN, FAAN \\ Francis Yang, PhD \\ University of Kansas School of Nursing
}

ndrogen deprivation therapy (ADT) is the standard treatment for metastatic
hormone responsive prostate cancer $(\mathrm{CaP})$, and when surgery or radiation is
not an option for localized $\mathrm{CaP}$. ADT is increasingly recommended as an adjuvant treatment with radiation therapy ${ }^{1}$ because of its survival benefit. ${ }^{2}$ ADT has also been shown to benefit men with metastatic disease. It can delay the onset of symptoms such as pain and fracture from bone metastasis, urinary obstruction, and bowel obstructions. ${ }^{3}$ ADT can be effective for two to three years in delaying the progression of $\mathrm{CaP}$ and its symptoms. Yet, it has been demonstrated that Black men are less likely to receive ADT compared to their White counterparts $(\mathrm{OR}=0.64) .{ }^{4-6}$ In a previous study using SEER-Medicare linked data, among men with locoregional CaP, Black men were significantly less likely (24\%) to receive ADT than other White men. ${ }^{6}$ Furthermore, Cobran and colleagues (2018) found that Black men with metastatic CaP experienced significantly delayed receipt of ADT as compared to White men (172 days vs. 95 days, $\mathrm{p}<0.05){ }^{7}$ Additionally, others using SEER-Medicare data found that Black men with metastatic $\mathrm{CaP}$ were less likely to receive ADT, and when they did receive it, the treatment was delayed compared to other men., Despite the evidence supporting the benefit of ADT, in combination with radiation therapy, either adjuvantly or neoadjuvantly, to produce better outcomes, ${ }^{9}$ Black men are less likely to receive ADT with radiation therapy. Findings from a population-based study of men with locally advanced CaP showed that, even though radiation therapy combined with ADT is better than either alone, only $8 \%$ of Black men received radiation therapy plus ADT compared to $84 \%$ of other men. ${ }^{10}$ Therefore, the purpose of this convergent, mixed methods study is to explore provider and patient factors related to ADT receipt between Black and White men in a midwestern health system.

Specifically, we aim to:

1. Describe differences in ADT receipt between Black men and White men using the EPIC Clarity database, which includes clinical and demographic data by:

a. Adjuvant use with radiation therapy;

b. Timing to initiation of ADT with metastatic $\mathrm{CaP}$; and

c. Type of ADT utilized (surgical vs. medical).
2. Identify provider characteristics (e.g., specialty, years in practice, age, gender) and ADT utilization/ recommendation patterns for Black men and White men.

3. Explore perceptions of and experiences with ADT among Black and White men using individual, semi-structured interviews.

From our analyses of these data we will create an initial explanatory framework of differences in the receipt of ADT 
between Black men and White men, which will be the foundation for future testing and eventual development of practice guidelines and policy recommendations.

\section{Background}

Black men are diagnosed with more advanced and aggressive $\mathrm{CaP}$ at an earlier age than men of other ethnicities and are $44 \%$ to $75 \%$ more likely to develop metastases. ${ }^{11,12}$ While overall mortality rates from $\mathrm{CaP}$ have declined, they have not declined as rapidly for Black men, and studies still indicate that Black men are more likely to receive inferior treatment. ${ }^{11,13-16}$

Factors influencing ADT decision-making that underlie treatment patterns are complex and include patient, provider, system, and socio-cultural factors, as well as social determinants of health $(\mathrm{SDOH})$. Much of the literature on $\mathrm{CaP}$ patient decision-making is focused on screening and early-stage treatment (prostatectomy, radiation treatment, or active surveillance), and decision aids (DA) ${ }^{17-23}$ while race/ethnicity's role in $\mathrm{CaP}$ decision-making has not been extensively explored. Some factors found to influence men's treatment decisions are treatment-related side effects, such as erectile dysfunction, urinary dysfunction, bowel dysfunction, quality of life (QOL), and, most strongly, physician recommendation. ${ }^{5,17}$ A few studies have addressed race in treatment decision-making, primarily in screening decisions and DA for early-stage $\mathrm{CaP} .{ }^{24-27}$ One study was found to address patient perceptions toward recurrent $\mathrm{CaP}$ and $\mathrm{ADT}$, but it only focused on outcomes of a DA, and did not address race/ethnicity..$^{25}$ Thus, there is a gap in the literature regarding the factors contributing to differences in ADT receipt between Black and White men.

In early-stage $\mathrm{CaP}$ treatment deci- sion-making, the literature demonstrates that providers are influenced by grade, survival prediction, and patient financial and insurance statuses. ${ }^{28,29}$ There is a growing body of research studying the effect of implicit bias on the provider's clinical decision-making, with providers having a White preference, regardless of the provider's specialty. ${ }^{30-37}$ Most of the studies used the Implicit Bias Association Test (IAT) or vignette design to examine the influence of patient characteristics on treatment decisions. Another body of literature supports the practice of shared decision-making. ${ }^{38,39}$ While patient characteristics related to the desire for shared decision-making were explored, provider characteristics related to approach to and acceptance of shared decision-making was not. Provider characteristics were addressed to some extent in the literature on burnout, depression, and physician engagement. ${ }^{40-45}$ One study, conducted in Ireland, found that as the provider age increased and socio-economic status of the patients decreased, quality of care decreased. ${ }^{46}$ Thus, provider characteristics can play an important and sometimes unrecognized role in treatment recommendations, including those related to ADT.

Numerous studies investigating ADT have not identified race in their samples, and if they did, race was not included in analyses. ${ }^{47-49}$ While some documentation of differences in ADT receipt by Black men compared to White men exists as noted above, there is a dearth of literature exploring factors that may explain these differences, specific to ADT, from either the provider characteristics or the patient perspectives. This study will lay the foundation for future interventions to address disparities in ADT receipt by Black men.

We will use the National Institute on Minority Health and Health Disparities 
Table 1. Adapted NIMHD Research Framework for ADT Utilization

\begin{tabular}{|l|l|l|l|l|}
\hline & Individual & Interpersonal & Community & Social \\
\hline Biologic & $\begin{array}{l}\text { CaP stage } \\
\text { Comorbidities } \\
\text { Age (Aim 2,3) }\end{array}$ & & & \\
\hline Behavioral & $\begin{array}{l}\text { Men's perceptions } \\
\text { Information-seeking } \\
\text { Care-seeking (Aim 3) }\end{array}$ & & & \\
\hline $\begin{array}{l}\text { Sociocultural } \\
\text { Environment }\end{array}$ & $\begin{array}{l}\text { Race } \\
\text { Income/occupation } \\
\text { Education } \\
\text { (Aim 2.3) }\end{array}$ & Partnership status (Aim 3) & $\begin{array}{l}\text { Men's experienc- } \\
\text { es in healthcare } \\
\text { systems (Aim 3) }\end{array}$ & \\
\hline $\begin{array}{l}\text { Healthcare } \\
\text { System }\end{array}$ & $\begin{array}{l}\text { Insurance coverage } \\
\text { (Aim 2,3) } \\
\text { Barriers (Aim 3) }\end{array}$ & $\begin{array}{l}\text { Patient-provider relation- } \\
\text { ship (Aim 3) } \\
\text { Provider characteristics } \\
\text { (Aim 2,3) } \\
\text { ADT prescription patterns } \\
\text { (Aim 1,2) }\end{array}$ & & $\begin{array}{l}\text { Differences in } \\
\text { ADT receipt by } \\
\text { Black men } \\
\text { (Aim 1) }\end{array}$ \\
& & & & \\
& & & \\
\end{tabular}

Explanatory Framework of Differences in ADT Receipt for Black Men

(NIMHD) Research Framework, focusing on the individual and interpersonal levels of influence within the behavioral, sociocultural and healthcare system domains of health. Outcomes explored in this study include individual health and population health, with a description of factors that influence ADT utilization and decision-making for Black men who would benefit from ADT. We hypothesize that ADT utilization and decision-making will be different for Black men relative to White men. Table 1 illustrates the Domains and Levels to be explored in this study.

\section{Methods and Design}

We are using a convergent, mixed methods design to describe factors that contribute to differences in ADT receipt between Black men and White men.

For Aim 1, we are describing and comparing ADT utilization between Black men and White men. Receipt patterns are being explored for adjuvant use of ADT with radiation therapy, those with a diagnosis of hormone-responsive $\mathrm{CaP}$, and by type of ADT used. Type may be surgical (orchiectomy) or medical. While surgical ADT is no longer in common use, when it is used, there is a higher representation of underserved, minority men.

In Aim 2, we are exploring the characteristics of providers who prescribe ADT in an effort to describe patterns of ADT receipt by patient sociodemographics (e.g., age, insurance status, zip code), to uncover factors which may influence provider decision-making.

For Aim 3, we will examine Black and White men's perceptions of and experiences with ADT, including the men's interactions with providers about ADT. We will explore men's beliefs, attitudes, information received, and treatment options presented to them.

\section{Sample and Setting}

Aim 1: The University of Kansas Medical Center (KUMC) is associated with The University of Kansas Health System (TUKHS). TUKHS utilizes the Epic electronic health record (EHR) system, Epic Systems, Verona, WI. The EHR has been branded as "O2" which stands for Optimal Outcomes. Epic Clarity is a large relational database that receives a subset of data from $\mathrm{O} 2$ on a nightly basis and is typically used for operational and research reporting purposes. Inquiry 
within the Epic Clarity database identified approximately 14,495 men with $\mathrm{CaP}$ who were treated with ADT or who were candidates for ADT between 2010 and 2020.

Extracts will include candidates for ADT as defined as those with a diagnosis of metastatic CaP (APR-DRG, ICD-9, ICD-10) and the patient demographics (age, gender, race, ethnicity, date of diagnosis) will be collected. Among those men, we will identify those who had any combination of adjuvant ADT treatments, including those with radiation (using CPT codes), medical hormone-responsive ADT (using specific medications), and those who had surgical ADT (using CPT codes). Clarity captures the "authorizing" provider with a date/time stamp for every diagnosis documented and orders for ADT treatments. Provider demographics such as name and the National Provider Identifier (NPI) from the Clarity data will be the common link to additional data sources.

Aim 2: Using the provider name and NPI, we will extract provider demographics obtained from the Human Resource systems (Kronos and Workday) and/or the National Plan \& Provider Enumeration System (NPPES). The integrated data will be used to compare and contrast patterns in treatment regimens. ADT is prescribed by oncologists, radiation oncologists, and urologists at the TUKHS. There are currently 51 oncologists, 16 radiation oncologists, and 15 urologists practicing.

ADT prescribing patterns will be obtained from the Clarity data and matched to provider characteristics obtained from the Kronos, Workday, and/or the NPPES dataset. The unique NPI is the key to integrating the datasets. The integration of various data sources enables researchers to look at the data in new ways that are not available when viewing data one source at a time.

Aim 3: We will recruit a stratified purposive sample of Black and White men from those identified as eligible for ADT in Aim 1. Using a stratified sampling approach will facilitate comparisons based on self-identified race. Eligibility for inclusion in the interviews: 1 . Aged 45 or older, 2. Self-identified as non-Hispanic Black or White, and 3. Willing and able to articulate perceptions and experiences about ADT. We will purposively select men who received ADT and men who were candidates for ADT (as defined above) but did not receive ADT. We will recruit and collect data until we reach data saturation to allow for comparisons between groups. However, based on qualitative literature, ${ }^{50}$ data saturation can be reached with 15-30 participants, so we plan to include a sample of 30 men: 15 men who identify as Black and 15 men who identify as White.

The Patient Identification via Pioneers Registry is a registry of patients in the community who are interested in research; the registry will be used to invite people to participate. We will work with established community contacts in the Black community and a prostate cancer support group to create a Patient Advisory Board (PAB). The PAB will provide advisement on best methods for study recruitment and enrollment, the development of interview guides, and the interpretation of qualitative data. Virtual meetings will be scheduled quarterly, with communications with the study team in between as needed. Semi-structured interviews will be conducted using a HIPAA-compliant virtual platform.

Data Collection Schedule and Procedures

The data collection workflow and data sources are outlined in Figure 1. 


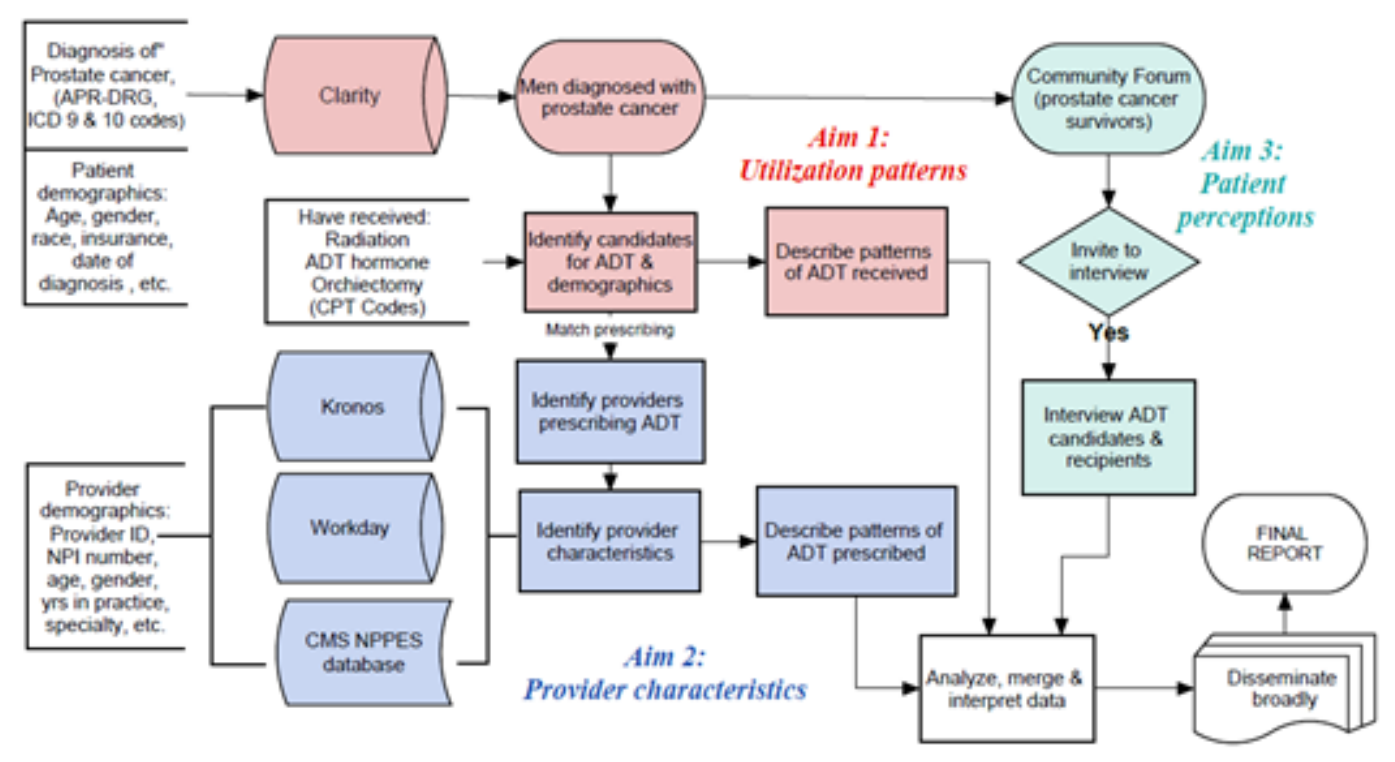

Figure 1. Data collection workflow.

Aim 1: First, data of men who were candidates for ADT will be extracted from the Clarity database. Next, they will be grouped by those who did and did not receive ADT by race/ethnicity. Those who received $\mathrm{ADT}$ will be grouped by type of ADT (surgical vs. medical), CaP type (adjuvant or metastatic recurrent disease), and by race/ethnicity. Next, for the men with metastatic disease, we will identify the time from receipt of metastatic diagnosis to initiation of ADT. Men on ADT will be identified using APR-DRG, ICD10 designation for $\mathrm{CaP}$. A thorough and complete search for surgical and medical ADT will be completed using the following search terms: orchiectomy (surgical ADT); luteinizing hormone-releasing hormone (LHRH) agonists, which include Leuprolide (Lupron, Eligard), Triptorelin (Trelstar), Goserelin (Zoladex), Histrelin (Vantas); LHRH antagonists, which include Degarelix (Firmagon); and anti-androgen therapy, which includes Flutamide (Eulexin), Bicalutamide (Casodex), Nilutamide (Nilandron), Abiraterone (Zytiga), and Ketoconazole (Nizoral). Anti-androgens, which can be used for castrate-resistant $\mathrm{CaP}$ (for those who are no longer responding to other forms of hormone therapy), include Enzalutamide (Xtandi), Darolutamide (Nubeqa), and Apalutamide (Erleada).

Aim 2: To describe provider characteristics and prescribing patterns, we will manually retrieve NPPES data, using the NPI as key. This will allow us to investigate whether clusters of specific provider characteristics correspond with ADT prescribing patterns for Black men compared with White men. Provider characteristics will include age, time in practice, medical education in the U.S. or international, certification, location (urban, rural), and specialty.

Aim 3: Before recruiting for the individual interviews, we will establish the PAB, which will be comprised of Black and White CaP survivors. We will work with an African American Cancer Community Advisory Board, co-led by Mr. Broderick Crawford and Dr. Peltzer, and the Prostate Cancer Network, led by Mr. Steve Hernsten, to identify and invite six men to be a part of the PAB. The PAB will be convened to provide insights 
into perceptions of men relative to $\mathrm{CaP}$ and ADT. The PAB will help develop the semi-structured interview guide to ensure the questions are culturally appropriate and sensitive for eliciting men's understanding and perceptions of $\mathrm{CaP}$ and ADT, factors that influenced their receipt of ADT, sources of information, and their perceptions of their interactions with their provider regarding ADT.

The PAB will also assist with recruitment efforts, creating recruitment materials and identifying the best approaches (mailings, social media, telephone calls) for inviting potential participants and disseminating study information through their social networks. The recruitment materials will also be shared with clinic staff to disseminate and by study staff via email, telephone, or mail. Only men who have given permission to be contacted for research in the EHR will be contacted directly by study staff. The information will include the contact information of the study coordinator to further information and interest in the study. The study coordinator will respond to contacts and call to provide further information, ascertain eligibility, and invite participation.

IRB-approved online consent will be obtained and an interview appointment made. Interviews will be conducted by videoconference, if possible, and by phone, if videoconference is not available. Videoconference is preferable because visual cues, such as facial expression, are visible. However, our previous work has demonstrated that rich data can be obtained by phone interviews with careful attention to voice tone, cadence, and background noise. Interviews are expected to last 45-60 minutes and will be audio recorded. The audio recordings will be transcribed verbatim in preparation for data analysis. Participants will receive $\$ 50$ compensation for their time.
The interviews will be conducted by a member of the research team who will be trained in qualitative interview techniques by the PI (Maliski) and Co-I (Peltzer). Prior to beginning data collection, the interviewer(s) will conduct two simulated interviews with Dr. Maliski or Peltzer, who will provide feedback on the technique. The interviews will begin with an overview of the study, including the purpose and a reminder that participation is voluntary, confidentiality will be maintained, and if there are any questions that are uncomfortable, the participant does not have to answer. The interviewer will ask for the study participant to share a little bit about his general health, $\mathrm{CaP}$ history, and other comorbidities before transitioning to questions about ADT. Following the completion of the interview, sociodemographic data, including age, self-reported race, insurance status, income range, employment, partnership status, and education, will be collected.

\section{Data Analysis, Merging, and Interpretation}

The culmination of the analyses will be an integration of the aims to produce an explanatory description of ADT utilization disparities and associated factors.

Aim 1: Length of time from diagnosis of metastatic CaP to initiation of ADT for each group (Black vs. White) will be compared, using the Kaplan-Meier estimator, represented through the Kaplan-Meier curve to show the probability of treatment from time zero diagnosis over fiveyear intervals. The test for proportional assumptions, using the scaled Schoenfeld residuals and Kaplan-Meier curves, will be satisfied before proceeding to the Cox Proportional Hazards Regression Model. A Cox Proportional Hazards Regression Model will be used to examine if there are statistically significant differences in surgical and medical ADT and receipt 
of adjuvant ADT with radiation therapy between Black men and White men, and types and numbers of comorbidities experienced before and after treatment between groups over time, respectively.

Aim 2: To identify the profiles of provider characteristics [age, time in practice, certification, location (urban, rural), medical education (U.S. or international), specialty, and ADT prescribing patterns], a latent class analysis will be used to identify the best fitting number and type of clusters within the Clarity dataset. Latent class analysis is based on posterior probabilities estimated through bootstrapping the data over multiple iterations to identify the patterns of characteristics that best describe data that may not emerge through traditional statistical models. The goodness-of-fit statistics are as follows: Akaike Information Criterion (AIC), Log-likelihood (LL), Bayesian Information Criterion (BIC), and sample size-adjusted BIC (SABIC). ${ }^{51}$ In Mplus version $8.39^{52}$ software, bootstrap likelihood ratio test (LRT) and Vuong-LoMendell-Rubin LRT to determine the best-fitting model between two sequential LCA estimated models, with a higher p-value favoring the prior LCA model.

Aim 3: Data will be analyzed concurrently with data collection using qualitative content analysis. ${ }^{53}$ This will allow emergent themes to shape subsequent data collection to more fully describe categories. Data will be entered into DeDoose for data management. Analyses will be conducted by the PI (Maliski) and co-I (Peltzer). Both will independently read the initial five transcripts and code using segment-by-segment coding to capture the main thought in each segment. They will then cluster codes into broader categories after which the analysts will meet to develop consensus on the codes and categories. Using those categories, the analysts will code transcripts as the data are collected. New codes or categories will be added as they emerge. Analysts will meet to review data and categories, returning to the data to validate categories. The PAB will be consulted regarding the relevance of the categories. Finally, from the categories, analysts will develop themes that represent the data and describe men's perceptions of and experience with ADT and interactions with providers.

To ensure rigor, the research team will maintain a coding manual. Drs. Maliski and Peltzer will create, maintain, update, and revise the list of codes. Drs. Maliski and Peltzer will maintain at least $80 \%$ interpretive convergence. ${ }^{54}$ The research team will also maintain analytic memos to document their coding processes, choices of codes, and the emergent categories, themes, and patterns. ${ }^{54}$ The analytic memos will also be used to reflect on and document any problems that may transpire with the study and to assist with the final report for the study. The analytic memos, meeting minutes, and codebook will be used as the audit trail to ensure credibility of the study. The analysts will also complete member checking with a subsample of participants and provide the emerging themes to the $\mathrm{PAB}$ as an additional method of credibility and confirmability. We will carefully evaluate the emerging themes for differences between Black and White men regarding their perceptions and experiences.

Merging of Data: After statistical analysis of quantitative data and content analysis of interviews, the data will be merged for analysis. We will examine the textual data from the interviews and compare to ADT receipt patterns and to the provider characteristics and prescribing patterns. We will also assess the fit 
of integration of the data ${ }^{55}$ to evaluate if there is confirmation of or discordance between the men's perceptions and experiences with differences of ADT receipt patterns. If there is confirmation, the in- tegrated model will provide a comprehensive description of factors related to differences in ADT receipt between Black men and White men.

\section{References}

1. Shevach, J., Chaudhuri, P., \& Morgans, A. K. (2019). Adjuvant therapy in high-risk prostate cancer. Clin Adv Hematol Oncol, 17(1):45-53.

2. Kovtun, K., Chen, M., Braccioforte, M., Moran, B., \& D'Amico, A. (2016). Race and mortality risk after radiation therapy in men treated with or without androgen supression therapy for favorable-risk prostate cancer. Cancer, 122:3608-3614.

3. Carson, A. P., Howard, D. L., Carpenter, W. R., et al. (2010). Trends and racial differences in the use of androgen deprivation therapy for metastatic prostate cancer. J Pain Symptom Manage, 39(5):872-881.

4. Nguyen, C., Lairson, D. R., Swartz, M. D., \& Du, X. L. (2019). Racial, Socioeconomic, and Geographic Disparities in the Receipt, Timing to Initiation, and Duration of Adjuvant Androgen Deprivation Therapy in Men with Prostate Cancer. J Racial Ethn Health Disparities, 6(1):133-142.

5. Holmes-Rovner, M., Montgomery, J. S., Rovner, D. R., et al. (2015). Informed Decision Making: Assessment of the Quality of Physician Communication about Prostate Cancer Diagnosis and Treatment. Med Decis Making, 35(8):999-1009.

6. Holmes, L., Chan, W., Jiang, Z., Ward, D., Essien, E. J., \& Du, X. L. (2009). Impact of Androgen Deprivation Therapy on Racial/Ethnic Disparities in the Survival of Older Men Treated for Locoregional Prostate Cancer. Cancer Control, 16(2):176-185.

7. Cobran, E. K., Young, H. N., Chen, R. C., et al. (2019). Race and Time to Receipt of Androgen Deprivation Therapy Among Men With Metastatic Prostate Cancer. J Natl Med Assoc, 111(3):246-255.

8. Keating, N. L., O'Malley, A. J., McNaughton-Collins, M., Oh, W. K., \& Smith, M. R. (2008). Use of androgen deprivation therapy for metastatic prostate cancer in older men. BJU Int, 101(9):1077-1083.

9. Cerne, J. Z., McGuire, S. E., Grant, S. R., et al. (2013). Factors associated with improved biochemical response to neoadjuvant androgen deprivation therapy before definitive radiation therapy in prostate cancer patients. Prostate Cancer Prostatic Dis, 16(4):346-351.

10. Lowrance, W. T., Elkin, E. B., Yee, D. S., et al. (2012). Locally advanced prostate cancer: a population-based study of treatment patterns. BJU International, 109(9):13091314.

11. Miller, E., Pinsky, P., Black, A., Andriole, G., \& Pierre-Victor, D. (2018). Secondary prostate cancer screening outcomes by race in the Prostate, Lung, Colorectal, and Ovarian (PLCO) screening trial. Prostate, 78:830-838.

12. Tsodikov, A., Gulati, R., \& de Carvalho, T. (2017). Is prostate cancer different in black men? Answers from 3 natural history models. Cancer, 123:2312-2319.

13. Lee, D., Barocas, D., \& Zhao, Z. (2018). Contemproary prostate cancer radiation therapy in the United States: patterns of care and compliance with quality measures. Practical Radiation Oncology, 2018(8):307-316. 
14. Cobran, E., Young, H., Chen, R., et al. (2018). Race and time to receipt of androgen deprivation therapy among men with metastatic prostate cancer. Journal of the $\mathrm{Na}$ tional Medical Association, in press.

15. Cobran, E., Chen, R., Overnam, R., et al. (2016). Racial differences in diffusion of intensity-modulated radiation therapy for localized prostate cancer. American Journal of Men's Health, 10(5):399-407.

16. Moses, K., Paciorek, A., Penson, D., Carroll, P., \& Master, V. (2010). Impact of ethnicity on primary treatment choice and mortality in men with prostate cancer: Data from CaPSURE. Journal of Clinical Oncology, 28(6):1069-1074.

17. Thera, R., Carr, D. T., Groot, D. G., Baba, N., \& Jana, D. K. (2018). Understanding Medical Decision-making in Prostate Cancer Care. Am J Mens Health, 12(5):16351647.

18. Dierks, T., Heijnsdijk, E. A. M., Korfage, I. J., Roobol, M. J., \& de Koning, H. J. (2019). Informed decision-making based on a leaflet in the context of prostate cancer screening. Patient Educ Couns, 102(8):1483-1489.

19. Martínez-González, N. A., Plate, A., Markun, S., Senn, O., Rosemann, T., \& Neuner-Jehle, S. (2019). Shared decision making for men facing prostate cancer treatment: a systematic review of randomized controlled trials. Patient Prefer Adherence, 13:1153-1174.

20. Sandiford, L., \& D'Errico, E. M. (2016). Facilitating Shared Decision Making About Prostate Cancer Screening Among African American Men. Oncol Nurs Forum, 43(1):86-92.

21. Gorawara-Bhat, R., O'Muircheartaigh, S., Mohile, S., \& Dale, W. (2017). Patients' perceptions and attitudes on recurrent prostate cancer and hormone therapy: Qualitative comparison between decision-aid and control groups. J Geriatr Oncol, 8(5):368-373.

22. Pacyna, J. E., Kim, S., Yost, K., et al. (2018). The comparative effectiveness of decision aids in diverse populations with early stage prostate cancer: a study protocol for a cluster-randomized controlled trial in the NCI Community Oncology Research Program (NCORP), Alliance A191402CD. BMC Cancer, 18(1):788.

23. Jalil, N. B., Lee, P. Y., Nor Afiah, M. Z., et al. (2020). Effectiveness of Decision Aid in Men with Localized Prostate Cancer: a Multicenter Randomized Controlled Trial at Tertiary Referral Hospitals in an Asia Pacific Country. J Cancer Educ.

24. Owens, O. L., Felder, T., Tavakoli, A. S., et al. (2019). Evaluation of a Computer-Based Decision Aid for Promoting Informed Prostate Cancer Screening Decisions Among African American Men: iDecide. Am J Health Promot, 33(2):267-278.

25. Woods-Burnham, L., Stiel, L., Wilson, C., et al. (2018). Physician Consultations, Prostate Cancer Knowledge, and PSA Screening of African American Men in the Era of Shared Decision-Making. Am J Mens Health, 12(4):751-759.

26. Owens, O. L., Friedman, D. B., Brandt, H. M., Bernhardt, J. M., \& Hébert, J. R. (2016). Digital Solutions for Informed Decision Making: An Academic-Community Partnership for the Development of a Prostate Cancer Decision Aid for African American Men. Am J Mens Health, 10(3):207-219.

27. Trinh, Q. D., Hong, F., Halpenny, B., Epstein, M., \& Berry, D. L. (2020). Racial/ ethnicity differences in endorsing influential factors for prostate cancer treatment choice: An analysis of data from the personal patient profile-prostate (P3P) I and II trials. Urol Oncol, 38(3):78.e77-78.e13. 
28. Thurtle, D., Rossi, S. H., Berry, B., Pharoah, P., \& Gnanapragasam, V. J. (2019). Models predicting survival to guide treatment decision-making in newly diagnosed primary non-metastatic prostate cancer: a systematic review. BMJ Open, 9(6):e029149.

29. Walker, D. M., McAlearney, A. S., Sova, L. N., Lin, J. J., Abramson, S., \& Bickell, N. A. (2016). Comparing Prostate Cancer Treatment Decision Making in a Resource-rich and a Resource-poor Environment: A Tale of two Hospitals. J Natl Med Assoc, 108(4):211-219.

30. FitzGerald, C., \& Hurst, S. (2017). Implicit bias in healthcare professionals: a systematic review. BMC Med Ethics, 18(1):19.

31. Maina, I. W., Belton, T. D., Ginzberg, S., Singh, A., \& Johnson, T. J. (2018). A decade of studying implicit racial/ethnic bias in healthcare providers using the implicit association test. Soc Sci Med, 199:219-229.

32. Hall, W. J., Chapman, M. V., Lee, K. M., et al. (2015). Implicit Racial/Ethnic Bias Among Health Care Professionals and Its Influence on Health Care Outcomes: A Systematic Review. Am J Public Health, 105(12):e60-76.

33. Dehon, E., Weiss, N., Jones, J., Faulconer, W., Hinton, E., \& Sterling, S. (2017). A Systematic Review of the Impact of Physician Implicit Racial Bias on Clinical Decision Making. Acad Emerg Med, 24(8):895-904.

34. Schnierle, J., Christian-Brathwaite, N., \& Louisias, M. (2019). Implicit Bias: What Every Pediatrician Should Know About the Effect of Bias on Health and Future Directions. Curr Probl Pediatr Adolesc Health Care, 49(2):34-44.

35. Balakrishnan, K., \& Arjmand, E. M. (2019). The Impact of Cognitive and Implicit Bias on Patient Safety and Quality. Otolaryngol Clin North Am, 52(1):35-46.

36. James, S. A. (2017). The strangest of all encounters: racial and ethnic discrimination in US health care. Cad Saude Publica, 33Suppl 1(Suppl 1):e00104416.

37. John-Henderson, N. A. (2015). Implicit Cognition: Implications for Global Health Disparities. Child Adolesc Psychiatr Clin N Am, 24(4):751-763.

38. Elwyn, G., Frosch, D. L., \& Kobrin, S. (2016). Implementing shared decision-making: consider all the consequences. Implement Sci, 11:114.

39. Gustin Jr., A. N. (2019). Shared Decision-Making. Anesthesiol Clin, 37(3):573-580.

40. Neugut, A. I., MacLean, S. A., Dai, W. F., \& Jacobson, J. S. (2019). Physician Characteristics and Decisions Regarding Cancer Screening: A Systematic Review. Popul Health Manag, 22(1):48-62.

41. Rotenstein, L. S., Torre, M., Ramos, M. A., et al. (2018). Prevalence of Burnout Among Physicians: A Systematic Review. Jama, 320(11):1131-1150.

42. Scholl, I., LaRussa, A., Hahlweg, P., Kobrin, S., \& Elwyn, G. (2018). Organizational- and system-level characteristics that influence implementation of shared decision-making and strategies to address them - a scoping review. Implement Sci, 13(1):40.

43. Perreira, T. A., Perrier, L., \& Prokopy, M. (2018). Hospital Physician Engagement: A Scoping Review. Med Care, 56(12):969-975.

44. Azam, K., Khan, A., \& Alam, M. T. (2017). Causes and Adverse Impact of Physician Burnout: A Systematic Review. J Coll Physicians Surg Pak, 27(8):495-501.

45. Shahbazi, S., \& Woods, S. J. (2016). Influence of physician, patient, and health care system characteristics on the use of outpatient mastectomy. Am J Surg, 211(4):802809. 
46. Riordan, F., McHugh, S. M., O’Donovan, C., Mtshede, M. N., \& Kearney, P. M. (2020). The Role of Physician and Practice Characteristics in the Quality of Diabetes Management in Primary Care: Systematic Review and Meta-analysis. J Gen Intern Med, 35(6):1836-1848.

47. Shibata, Y., Suzuki, K., Arai, S., et al. (2013). Impact of pre-treatment prostate tissue androgen content on the prediction of castration-resistant prostate cancer development in patients treated with primary androgen deprivation therapy. Andrology, 1(3):505-511.

48. Demir, A., Cecen, K., Karadag, M. A., Kocaaslan, R., \& Turkeri, L. (2014). The course of metastatic prostate cancer under treatment. Springerplus, 3:725.

49. Moul, J. W. (2015). Hormone naïve prostate cancer: predicting and maximizing response intervals. Asian J Androl, 17(6):929-935; discussion 933.

50. Creswell, J. W., \& Creswell, D. J. (2018). Research Design: Qualitative, Quantitative, and Mixed Methods Approaches. Sage Publications.

51. McCutcheon, A. (2002). Latent Class Analysis, Vol 64. Newbury Park: SAGE Publications.

52. Muthén, L. K, \& Muthén, B. O. (1998-2020). Mplus User's Guide, Eighth ed. Los Angeles, CA: Muthén \& Muthén.

53. Sandelowski, M. (2000). Whatever happened to qualitative description? Research in Nursing \& Health, 23(4):334-340.

54. Saldana, J. (2009). The coding manual for qualitative researchers. Sage Publications Ltd.

55. Fetters, M. D., Curry, L. A., \& Creswell, J. W. (2013). Achieving integration in mixed methods designs-principles and practices. Health Serv Res, 48(6 Pt 2):2134-2156. 